\title{
Ampliação e flexibilização de horários na APS: análise das experiências do Prêmio APS Forte
}

\author{
Erika Rodrigues de Almeida e Francy Webster de Andrade Pereira
}

\section{RESUMO}

Este artigo apresenta uma análise das experiências do Prêmio APS Forte relacionadas à ampliação e flexibilização de horários, sendo a maioria com resultados positivos na ampliação do acesso, principalmente para pessoas que nunca tinham frequentado os serviços da APS, com aumento de atendimentos/procedimentos e resolutividade (especialmente pela ampliação da oferta de atendimentos à demanda espontânea, o que também produziu efeitos relacionados à redução de encaminhamentos), à melhoria do processo de trabalho das equipes e da interação dos trabalhadores com a comunidade, além de maior satisfação dos usuários.

Palavras-chave: Atenção Primária à Saúde; Acesso; Prêmio APS.

Revista da Rede APS 2020

Publicada em: 04/09/2020

DOI:10.14295/aps.v2i3.147

Erika Rodrigues de Almeida (Ministério da Saúde, Brasil)

Francy Webster de Andrade Pereira

(Ministério da Saúde, Brasil)

\section{ABSTRACT}

Correspondência para:

This article analyses the experiences of the PHC Award related to the expansion and flexibility of schedules, most of them with positive results in the expansion of access, Erika Rodrigues de Almeida especially for people who had never attended the PHC services, with an increase in attendance/procedures and resolutiveness (especially by the expansion of the offer of spontaneous demand, which also produced effects related to the reduction of referrals), the improvement of the work process of the teams and the interaction of the workers with the community, in addition to greater user satisfaction.

Keywords: Prmary Health Care; Access; PHC Prize. 


\section{AMPLIAÇÃO E FLEXIBILIZAÇÃO DE HORÁRIOS NA APS: ANÁLISE DAS EXPERIÊNCIAS DO PRÊMIO APS FORTE}

A Política Nacional de Atenção Básica define que a Atenção Básica seja a principal porta de entrada e centro de comunicação da Rede de Atenção à Saúde, coordenadora do cuidado e ordenadora das ações e serviços disponibilizados na rede. Define, ainda, que é responsabilidade tripartite assegurar ao usuário o acesso universal, equânime e ordenado às ações e serviços de saúde do SUS.O processo de trabalho, a combinação das jornadas de trabalho dos profissionais das equipes e os horários e dias de funcionamento devem ser organizados de modo que garantam amplamente acesso, o vínculo entre as pessoas e profissionais, a continuidade, coordenação e longitudinalidade do cuidado como princípios essenciais(BRASIL, 2017)

Starfield (2002) definiu quatro atributos essenciais da Atenção Primária à Saúde (APS): acesso de primeiro contato do indivíduo com o sistema de saúde, longitudinalidade, integralidade da atenção e coordenação da atenção dentro do sistema. Estabeleceu, ainda, três atributos derivados: atenção à saúde centrada na família, orientação comunitária e competência cultural. Ao apresentar os atributos essenciais, um serviço de saúde pode ser considerado provedor de APS, e essa atenção terá maior força se os atributos derivados também estiverem presentes. A autora ressalta que o acesso pode estar atrelado a diversas possibilidades de adentrar o serviço de saúde, dentre os quais estão a localização e a disponibilidade de horários e dias de funcionamento da unidade de saúde. Diversos estudos têm reportado a existência de barreiras de acesso aos serviços de Atenção Primária à Saúde (APS), dentre os quais se destacam o horário de atendimento insatisfatório durante o dia e a falta de atendimento no turno da noite e nos finais de semana (VEIRA LIMA et al.,2015; ESCOREL et al., 2007; PAULA et al., 2016;
CUNHA; VIEIRA-DA-SILVA, 2010; TURCI; LIMACOSTA; MACINKO, 2015).

As experiências do Prêmio APS Forte relacionadas ao subtema Ampliação $e$ flexibilização de horários somam 47 textos, produzidos por Equipes de Saúde da Família $(n=30)$, Secretarias Municipais de Saúde $(n=10)$, Coordenações ou Gerências de APS ( $n=4)$, Nasf AB $(n=1)$ e outros $(n=2)$. Regionalmente, predominam experiências do Sudeste $(n=16)$ e do Sul $(n=13)$, seguidas pelas regiões Nordeste $(n=9)$, Centro-Oeste $(n=6)$ e Norte $(n=3)$, com 9 (19\%) experiências realizadas em capitais. A maioria das experiências encontra-se em estágio avançado de execução $(n=38), 6 \mathrm{em}$ estágio inicial e 3 concluídas. Temporalmente, a maioria das experiências teve início de 2017 para cá (2017: $n=12 ; 2018: n=16 ; 2019: n=11)$.

A inovação das experiências aqui relatadas está na ampliação do horário de funcionamento das Unidades para além do horário comercial. Uma análise dos relatos permitiu identificar que os atributos do acesso/primeiro contato e da orientação comunitária embasaram a construção das experiências, visto que em sua maioria a motivação para instituir horários alternativos de funcionamento dos serviços de APS foi o reconhecimento de que os horários atuais de funcionamento - horário comercial estavam configurando barreira de acesso aos cuidados à população, em especial aos trabalhadores, trabalhadoras e homens que, em geral, buscam menos os serviços de saúde. Boa parte das experiências relata ter realizado planejamento prévio para definição do melhor horário de funcionamento das Unidades e/ou para definir a localização do serviço que passaria a funcionar com horário estendido. Em algumas experiências tal planejamento incluiu os usuários, além de gestores e trabalhadores das equipes. Todas optaram por ampliar o funcionamento dos serviços no horário do almoço e/ou à noite, outras ampliaram nos finais de semana. A maioria implantou tais mudanças em alguns dias da semana e/ou do mês, e organizou o serviço de modo a acolher tanto atendimentos de demanda espontânea quanto de demanda programada. Importante destacar que parte das experiências relatou ter 
sido necessário ampliar o quadro de recursos humanos e/ou remunerar horas extras e/ou permitir compensação das horas extras com folgas, de modo a não sobrecarregar os profissionais com extensiva carga horária laboral. Os dois principais referenciais apresentados pelas experiências foram: o modelo do Acesso Avançado (6 experiências relataram ter optado por este formato) e do Acolhimento com classificação/estratificação de risco para organização de agenda, ambos potenciais para a redução do absenteísmo, da demanda reprimida em extensas filas de espera e dos encaminhamentos aos serviços de urgência/emergência.

A maioria das experiências relatou resultados positivos no que tange à ampliação do acesso, sobretudo aos trabalhadores/trabalhadoras e a pessoas que nunca tinham frequentado os serviços da APS, à ampliação do número de atendimentos/procedimentos, ao aumento da resolutividade (especialmente pela ampliação da oferta de atendimentos à demanda espontânea, o que também produziu efeitos relacionados à redução de encaminhamentos), à melhoria do processo de trabalho das equipes e da interação dos trabalhadores com a comunidade, além de maior satisfação dos usuários.

Tais resultados corroboram com alguns achados da literatura, que reportam que a ampliação do horário de funcionamento dos serviços de APS favorece a ampliação do acesso aos cuidados em saúde à população, em especial aos trabalhadores e à população masculina. Adicionalmente, os estudos também observaram que a oferta de diversos serviços nos horários alternativos, especialmente a atenção à demanda espontânea, produz efeitos positivos na satisfação dos usuários e na redução de encaminhamentos (PAULA et al., 2016; CUNHA; VIEIRA-DA-SILVA, 2010; TURCI; LIMA-COSTA; MACINKO, 2015; ESCOREL et al., 2007). Por outro lado, a oferta de atendimento noturno restrita a apenas alguns serviços, como observado nas experiências aqui analisadas, pode limitar o acesso àqueles que residem com considerável distância geográfica, como relatado por Vieira-Lima et al. (2015). Assim, é fundamental que a definição dos locais de funcionamento dos serviços com horários alternativos seja realizada em conjunto com a população (nos municípios menores) e tenha certa capilarização (em grandes cidades), de forma que a localização seja estratégica e acessível para a maioria das pessoas.

A organização das agendas com base no Acesso Avançado dialoga com o estudo de Murray e Berwick (2003). Estes autores defendem que ao focar o atendimento nas demandas reais e do dia, as consultas se tornam mais objetivas (e mais rápidas) e produzem efeitos na redução dos encaminhamentos e da procura dos usuários por outros serviços de saúde. Além disso, tal método é capaz de produzir maior satisfação dos profissionais da APS, que passam a lidar com mais situações clínicas, favorecendo ampliação da resolutividade e maior satisfação dos usuários. Já no que diz respeito ao acolhimento com classificação/estratificação de risco, as experiências aqui analisadas dialogam com estudos de Mendes (2012) e Viegas e Penna (2012), que relacionam a redução das barreiras de acesso na APS por meio de práticas de acolhimento de qualidade, com diálogo aberto e escuta qualificada, e destacam a necessidade de instituição da classificação/estratificação de risco na APS para atendimento às urgências/emergências, de modo a aumentar o potencial resolutivo dos serviços.

Vale destacar que a PNAB prevê e recomenda que a unidade de saúde deve acolher todas as pessoas do seu território de referência, de modo universal e sem diferenciações excludentes. Destaca, ainda, que o atributo acesso tem relação com a capacidade do serviço em responder às necessidades de saúde da população (residente e itinerante), o que implica dizer que as necessidades desta devem ser o principal referencial para a definição do escopo de ações e serviços a serem ofertados, para a forma como esses serão organizados e para o todo o funcionamento da UBS, permitindo diferenciações de horário de atendimento (estendido, sábado, etc), formas de agendamento (por hora marcada, por telefone, e-mail, etc), e outros. Também recomenda evitar barreiras de acesso como o fechamento 
da unidade durante o horário de almoço ou em períodos de férias, entre outros, impedindo ou restringindo a acesso da população. Ressalta que horários alternativos de funcionamento que atendam expressamente a necessidade da população pode ser pactuada através das instâncias de participação social e gestão local (BRASIL, 2017).

Por fim, as experiências aqui analisadas dialogam com o recém lançado Programa Saúde na Hora (BRASIL, 2019), focado em ampliar o acesso aos serviços de APS por meio do funcionamento das Unidades de Saúde em horário estendido, com ampla carteira de serviços ofertada à população. $O$ programa também prevê maior acesso à Atenção Primária pelos usuários do SUS; mais ações de saúde em horários mais flexíveis para a população; ampliação da cobertura da Estratégia Saúde da Família e das equipes de Saúde Bucal nos municípios; fortalecimento da gestão municipal na organização da APS; redução de custos em outros níveis de atenção; redução de filas em unidades de pronto atendimento e emergências hospitalares. Vislumbra-se, assim, que muitas das experiências aqui relatadas são potenciais participantes do Programa, com possibilidade de ampliação das experiências em curso a partir da indução financeira federal para que as Unidades de Saúde possam funcionar 60 horas ou 75 horas semanais. 


\section{NOTAS E REFERÊNCIAS}

BRASIL. Ministério da Saúde. Portaria de Consolidação no 02, Anexo XXII, de 28 de setembro de 2017. Aprova a Política Nacional de Atenção Básica. Brasília: Ministério da Saúde, 2017.

BRASIL. Ministério da Saúde. Gabinete do Ministro. Portaria no 930, cria o Programa Saúde na Hora e dispõe sobre horário estendido de funcionamento em Unidade de Saúde da Família, maio de 2019.

BRASIL, Ministério da Saúde. Gabinete do Ministro. Portaria no 397, altera as Portarias de Consolidação no 2/GM/MS, de 28 de setembro de 2017, no 5/GM/MS de 28 de setembro de 2017, e n 6/GM/MS, de 28 de setembro de 2017, para dispor sobre o Programa Saúde na Hora, no âmbito da Política Nacional de Atenção Básica, março de 2020.

BRASIL, Ministério da Saúde. Gabinete do Ministro. Ministério, Portaria no 430, que estabelece incentivo financeiro ao funcionamento em horário estendido das Unidades de Saúde da Família (USF) ou Unidades Básicas de Saúde (UBS), considerando o cenário emergencial de saúde pública de importância internacional, março de 2020.

CUNHA, A. B. O.; VIEIRA-DA-SILVA, L. M. Acessibilidade aos serviços de saúde em um município do estado da Bahia, Brasil, em gestão plena do sistema. Cadernos de Saúde Pública, Rio de Janeiro, v. 26, n. 4, p 725737, abr. 2010.

WOLLMANN, A.; DA ROS, C.; LOWEN, I. M. V.; MOREIRA, L. R.; KAMI, M. T.; GOMES, M. A. G.; POLI NETO, P.Novas possibilidades de organizar o Acesso e a Agenda na Atenção Primária à Saúde. Curitiba, 2014.

ESCOREL, S.; GIOVANELLA, L.; MEDONÇA, M. H. M.; SENNA, M. C. M. O Programa de Saúde da Família e a construção de um novo modelo para a atenção básica no Brasil. Revista Panamericana de Salud Publica, Washington, v. 21, n. 2, p. 164-176, 2007.

PAULA, C. C.; SILVA, C. B.; TASSINARI, T. T.; PADOIN, S. M. M. Fatores que interferem no acesso de primeiro contato na atenção primária à saúde: revisão integrativa. Revista de Pesquisa: Cuidado é fundamental, Rio de Janeiro, v. 8, n. 1, p. 4056-4078, jan/mar. 2016.

STARFIELD, B. Atenção primária: equilíbrio entre necessidades de saúde, serviços e tecnologia. Brasília: UNESCO, Ministério da Saúde; 2002.

MENDES VILAÇA, E. O cuidado das condições crônicas na Atenção Primária à Saúde: o imperativo da consolidação da Estratégia da Saúde da Família. OPAS: Brasília, 2012.

MURRAY,M.;BERWICK,D.Advancedaccess: reducingwaitinganddelays in primarycare. EUA, Rev. JAMA, v.289, n.8, p: 1035-40, 2003.

TURCI, M. A.; LIMA-COSTA, M. F.; MACINKO, J. Influência de fatores estruturais e organizacionais no desempenho da atenção primária à saúde em Belo Horizonte, Minas Gerais, Brasil, na avaliação de gestores e enfermeiros. Cad. Saúde Pública, Rio de Janeiro, 31(9):1941-1952, set, 2015.

VIEGAS,S. M. F.; PENNA,C. M. M. O vínculo como diretriz para a construção da integralidade na Estratégia Saúde da Família. Rev Rede Enferm Nordeste. 2012;13(2):375-85.

VEIRA LIMA, S. A.; SILVA, M. R. F.; CARVALHO, E. M. F.; CESSE, E. A. P.; BRITO, E. S. V.; BRAGA, J. P. R. Elementos que influenciam o acesso à atenção primária na perspectiva dos profissionais e dos usuários de uma rede de serviços de saúde do Recife. Physis Revista de Saúde Coletiva, Rio de Janeiro, 25 [ 2 ]: 635 656, 2015. 